\title{
Actividad antihelmíntica in vivo de terpenos y aceites esenciales en pequeños rumiantes
}

\author{
Rafael Arturo Torres-Fajardo ${ }^{*} \otimes\left(\mathbb{D} ;\right.$; Rosa Isabel Higuera-Piedrahita2 ${ }^{\otimes(0)}$
}

\begin{abstract}
${ }^{1}$ Universidad Autónoma de Yucatán, Facultad de Medicina Veterinaria y Zootecnia, Yucatán, México. ¿Universidad Nacional Autónoma de México. Facultad de Estudios Superiores Cuautitlán. Cuautitlán. México. *Correspondencia: rafael-arturo-torres@outlook.es
\end{abstract}

Recibido: Marzo 2021; Aceptado: Junio 2021; Publicado: Junio 2021.

\section{RESUMEN}

Los terpenos y aceites esenciales (AcEs) poseen un amplio espectro de actividades biológicas que pueden ser exploradas en las ciencias veterinarias. En este sentido, su uso ha sido propuesto como una estrategia para enfrentar las crecientes poblaciones de nematodos gastrointestinales (NGI) resistentes a los antihelmínticos $(\mathrm{AH})$ tradicionales. En la presente revisión analizamos 11 manuscritos científicos que, mediante la utilización del enfoque in vivo, evaluaron el potencial AH de plantas ricas en terpenos (PRT) o AcEs en pequeños rumiantes. La especie ovina fue utilizada en el $81 \%$ de los trabajos. Brasil es el país que lidera esta línea de investigación seguido de los Estados Unidos de América y la República de Benín. Todos los manuscritos analizados utilizaron la prueba de reducción del conteo fecal de huevos de NGI, mientras que cinco manuscritos emplearon la metodología del test -o prueba - controlado(a). La actividad de las PRT y los AcEs sobre la excreción de huevos de NGI en las heces fue variable, reportándose valores que oscilaron desde un efecto nulo hasta un $97 \%$. Dos trabajos reportaron una reducción en el tamaño de los NGI machos adultos y en la fecundidad de las hembras tras la administración de AcEs. Resulta necesario generar más trabajos que se dirijan a entender las interacciones entre las plantas, sus metabolitos secundarios y los rumiantes que las consumen. Comprender dichas interacciones nos permitirá utilizar estos productos naturales como elementos que ayuden a mejorar la nutrición y la sanidad de ovinos y caprinos en diferentes sistemas productivos.

Palabras clave: Conteo de huevos de parásitos; enfermedades parasitarias; etnoveterinaria; nematodos gastrointestinales; cabras; metabolitos secundarios de plantas; ovejas (Fuentes: CAB, $\mathrm{MeSH})$.

\section{ABSTRACT}

Terpenes and essential oils (EsOi) have a wide range of biological activities that could be explored in veterinary sciences. In this sense, their use has been proposed as a strategy to overcome the growing populations of gastrointestinal nematodes (GIN) resistant to the traditional anthelmintic $(\mathrm{AH})$ drugs. In the present review, we analysed eleven scientific manuscripts aimed to evaluate the $\mathrm{AH}$ activity of terpene-rich plants (TRP) and EOs in small ruminants by using the in vivo approach. The sheep species was used in the $81 \%$ of works. Brazil is the country leading this research line,

Como citar (Vancouver).

Torres-Fajardo RA, Higuera-Piedrahita RI. Actividad antihelmíntica in vivo de terpenos y aceites esenciales en pequeños rumiantes. Rev MVZ Córdoba. 2021; 26(3):e2317. https://doi.org/10.21897/rmvz.2317 
followed by the United States of America and Benin. All the analysed manuscripts employed the faecal egg count reduction test, while five manuscripts employed the controlled test -or trialmethodology. The activity of TRP and EsOi on the faecal excretion of GIN eggs was variable, showing values between zero to $97 \%$. Two manuscripts reported a reduction in the size of male GIN, as well as the fecundity of female GIN after administration of EsOi. It would be worth to perform more works aimed to understand the relationships between plants, their secondary metabolites and the ruminants consuming them. Through the understanding of such interactions, we will be allowed to use these natural products as elements that could contribute to improve the nutrition and health of sheep and goats under different productive systems.

Keywords: Ethnoveterinary; gastrointestinal nematodes; goat; parasite egg count; parasitic diseases; plant secondary metabolites; sheep (Sources: $C A B, M e S H$ ).

\section{INTRODUCCIÓN}

\section{Control integrado de nematodos gastrointestinales en pequeños rumiantes}

Cualquier sistema de producción de ovinos y/o caprinos cuya fuente de alimentación esté basada en la utilización de pasturas se enfrenta invariablemente a la presencia de nematodos gastrointestinales (NGI). En la actualidad, la infección ocasionada por los NGI sigue siendo considerada como la mayor amenaza sanitaria para los rebaños de ovinos y caprinos, lo cual repercute negativamente en la viabilidad y la rentabilidad de dichos sistemas de producción $(1,2)$. Esta situación se ve potenciada por la aparición de cepas de NGI resistentes a los antihelmínticos $(\mathrm{AH})$ convencionales $(3,4)$ e incluso, a los principios activos de más reciente introducción en el mercado $(5,6,7,8)$. Se estima que las pérdidas ocasionadas por la infección con NGI y sus costos asociados son -en promedio- de 151, 206 y 86 millones de euros en ovinos lecheros, de carne y caprinos lecheros, respectivamente (9). Asimismo, en la Unión Europea se han estimado unas pérdidas aproximadas de 1.8 billones de euros como consecuencia de la resistencia a los AH (10). Con la intención de generar alternativas nofarmacológicas para hacer frente a los altos índices de resistencia de los NGI hacia los $A H$, se han propuesto diversas estrategias, las cuales se han encasillado bajo el término de control integrado de nematodos gastrointestinales. Este compendio de estrategias es de naturaleza complementaria (otorgando la posibilidad de adoptar varias a nivel de granja) y está dirigido a favorecer el control de las poblaciones de NGI por encima de su erradicación. Dentro de este abanico de estrategias se puede mencionar el uso racional de $\mathrm{AH}(11,12)$, la suplementación nutricional $(13,14)$, la selección genética $(15,16)$, la inmunización $(17,18)$, el uso de agujas de óxido de cobre $(19,20)$, la rotación de praderas $(21)$, el bio-control $(22,23)$ y el estudio de las propiedades $\mathrm{AH}$ de algunas especies de plantas $(24,25,26)$.

\section{Metabolitos secundarios de las plantas y sus propiedades antihelmínticas}

Invariablemente, todas las especies de plantas producen diferentes cantidades y concentraciones de ciertos elementos que poseen múltiples funciones biológicas documentadas hasta la fecha. Estos elementos han recibido diversas denominaciones en la literatura (27) tales como factores antinutricionales, compuestos secundarios de las plantas, metabolitos especializados o metabolitos secundarios de las plantas (MSP). Los MSP son compuestos químicos altamente especializados, producidos por las células de las plantas a través de diferentes vías metabólicas derivadas del metabolismo primario (28) e incluso su aparición se debe a las condiciones estresantes del medio ambiente, lo cual se ha denominado epigenética de plantas (29). La producción de MSP depende de la exposición de la planta a la luz, temperatura, estrés y características específicas del suelo como la salinidad y el $\mathrm{pH}(30,31)$.

En las ciencias veterinarias, la visión tradicional ha considerado a los MSP como sustancias producidas con la finalidad de controlar y repeler la herbivoría (32). No obstante, desde una perspectiva ecológica, la producción de MSP representa adaptaciones a situaciones específicas que mejoran la resiliencia, plasticidad y las interacciones entre las plantas y su ambiente $(33,34)$. Asimismo, es necesario considerar que el término herbívoro incluye algunas especies animales como los insectos, los cuales han coexistido con los MSP desde hace unos 350 
millones de años (35), o los rumiantes, cuyas familias ingresaron en la cronología de los ecosistemas hace unos 18-23 millones de años (36). Por lo tanto, las relaciones entre animales y MSP necesariamente involucran una respuesta adaptativa mutua. Como consecuencia, se ha documentado que el consumo de MSP puede representar beneficios de tipo sanitario mediante varios mecanismos, dentro de los que se destacan sus propiedades bioactivas contra los NGI de los pequeños rumiantes $(24,25,26)$. Actualmente se han identificado unos 200,000 MSP (37), aunque se cree que esta cifra podría representar una subestimación (38). Debido a la heterogeneidad en su composición química, existen muchos métodos de clasificación, sin embargo, de manera muy general se han establecido tres grandes grupos funcionales:

i.) Alcaloides: Con los cuales se dio el inicio formal al estudio de los MSP cuando el farmacéutico alemán Friedrich Sertürner aisló la morfina en el año 1806 (32). Esta familia de MSP posee unas 20,000 estructuras identificadas (39) y sus propiedades $\mathrm{AH}$ son materia actual de estudio $(40,41)$.

ii.) Compuestos fenólicos: De los cuales se han identificado 8,000 estructuras (42) y que representan el grupo de MSP más estudiados en lo que se refiere a las propiedades bioactivas en contra de los NGI en pequeños rumiantes. Dentro de este grupo, los taninos condensados han recibido una atención especial debido a su actividad $\mathrm{AH}$ y su presencia en varias especies de plantas presentes en sistemas de vegetación nativa a nivel mundial $(43,44,45)$.

iii.) Terpenos: Que representan el grupo más numeroso de MSP con unas 80,000 estructuras identificadas hasta la fecha (46) y cuya actividad $\mathrm{AH}$ es el motivo de la presente revisión.

\section{Terpenos, terpenoides e isoprenoides}

Estos MSP hacen parte del grupo más diverso y complejo desde el punto de vista químico y estructural. También hacen parte de esta numerosa familia los esteroides y carotenoides. Se estima que una tercera parte de los compuestos actualmente caracterizados en el diccionario de productos naturales (http://dnp.chemnetbase. com) pertenecen al denominado terpenoma. Este grupo de MSP está caracterizado por una gran diversidad, cuya estructura básica sigue el principio de las unidades de isopreno $\left(C_{5}\right)$ $\mathrm{n}$, o también conocido como 2-metil butano $\mathrm{y}$, que dependiendo de su número de subunidades $(n)$ se clasifican como hemiterpenos $\left(C_{5}\right)$, monoterpenos $\left(C_{10}\right)$, sesquiterpenos $\left(C_{15}\right)$, diterpenos $\left(C_{20}\right)$, sesterterpenos $\left(C_{25}\right)$, triterpenos $\left(\mathrm{C}_{30}\right)$ y tetraterpenos $\left(\mathrm{C}_{40}\right)(47)$. De manera general, los terpenos son sustancias volátiles mayormente responsables del olor de muchas plantas y frutas. El término terpeno proviene de un aceite volátil obtenido de algunas especies de pinos denominado trementina, cuya traducción al idioma inglés es turpentine (48). Debido a su amplia distribución en el reino vegetal, estos MSP pueden encontrarse en un vasto número de familias botánicas como Apiaceae, Asteraceae, Euphorbiaceae, Fabaceae, Lamiaceae, Myrtaceae, Poaceae, Rutaceae, Verbenaceae, entre muchas otras. Asimismo, son sintetizados por insectos y organismos marinos (49). Otro recordatorio importante es que, a diferencia de los terpenos, los terpenoides tienen añadido un grupo estructural en su composición química.

Los terpenos son altamente lipofílicos y volátiles en el medio ambiente, constituyendo una buena parte de los denominados compuestos orgánicos volátiles (COV) de las plantas. Los principales componentes de los COV son los monoterpenos y sesquiterpenos, seguidos de algunos compuestos con anillos aromáticos, ácidos grasos y aminoácidos volátiles (50). Por su parte, los diterpenos y triterpenos son menos volátiles. Los COV son utilizados por flores y frutos para enviar información a ciertas especies de animales, en especial insectos (51). Asimismo, se ha reportado que la acción de herbivoría de rumiantes sobre el follaje de varias especies de plantas libera monoterpenos y algunos sesquiterpenos en forma de COV (48).

Debido al creciente interés hacia los terpenos y los COV en diferentes actividades, ha sido necesario desarrollar metodologías que permitan obtener, aislar o purificar estos MSP. Estos procedimientos, en su mayoría basados en procesos químicos, han permitido el aislamiento y caracterización de algunos metabolitos secundarios. En este sentido, la síntesis de aceites esenciales (AcEs) representa una de las alternativas para obtener mezclas que son utilizadas en las industrias farmacéutica, agrícola y cosmética, entre otras.

\section{Aceites esenciales}

Definir con exactitud a los AcEs no es una tarea sencilla debido a la heterogeneidad en su composición química y a la multitud de 
propiedades que se les han atribuido en diversas actividades humanas como las industrias farmacéutica, cosmética, veterinaria y pecuaria. En la Tabla 1 se presentan algunas de las definiciones encontradas en la literatura. No obstante, se deben realizar algunas apreciaciones con la finalidad de clarificar este concepto: A pesar de su nombre, no son aceites (lípidos) verdaderos. De igual manera, el término esencial no se refiere a una propiedad de determinante, sino a que son derivados de componentes responsables de la fragancia o quinta essentia de las plantas (52), es decir, su nombre realmente es una derivación de la palabra esencia. Los AcEs pueden obtenerse de diversos recursos vegetales (hojas, flores, tallos, frutos, vainas, semillas, raíces y cortezas) o animales (organismos marinos) y representan mezclas lipofílicas que contienen una variedad de compuestos químicos (entre 20 y 60) obtenidos a partir de diversas metodologías (53). Por lo tanto, podríamos considerar a los AcEs como sustancias oleosas que contienen diferentes tipos de MSP, dentro de los cuales, los monoterpenos y sesquiterpenos constituyen una alta proporción. Finalmente, a pesar de que sus efectos bioactivos pueden ser atribuidos a los componentes mayoritarios, las sinergias entre diversos MSP deben ser consideradas y estudiadas.

Tabla 1. El concepto de aceite esencial de acuerdo con varios autores.

\begin{tabular}{|c|c|}
\hline Definición & Referencia \\
\hline $\begin{array}{l}\text { Mezclas de metabolitos secundarios obtenidas de la } \\
\text { fracción volátil de las plantas por destilación al vapor }\end{array}$ & (54) \\
\hline $\begin{array}{l}\text { Compuestos aromáticos ampliamente volátiles } \\
\text { extraídos de plantas por destilación al vapor o } \\
\text { extracción con solventes }\end{array}$ & (55) \\
\hline $\begin{array}{l}\text { Mezclas complejas de compuestos bioactivos } \\
\text { que presentan sustancias volátiles y lipofílicas } \\
\text { generalmente odoríferas y líquidas }\end{array}$ & (56) \\
\hline $\begin{array}{l}\text { Líquidos volátiles e hidrofóbicos extraídos de plantas, } \\
\text { generalmente ricos en aroma }\end{array}$ & (57) \\
\hline $\begin{array}{l}\text { Mezclas ampliamente heterogéneas de metabolitos } \\
\text { secundarios volátiles y aromáticos }\end{array}$ & $(58)$ \\
\hline
\end{tabular}

Como resultado de la búsqueda de estrategias no farmacológicas para enfrentar la resistencia $\mathrm{AH}$ y a los NGI, se han documentado las propiedades AH tanto de los terpenos $(59,60)$ como de los AcEs (61). Por lo tanto, el objetivo de la presente revisión será analizar los resultados de varios manuscritos científicos que, mediante la utilización del enfoque in vivo, evalúen la actividad $\mathrm{AH}$ de terpenos y AcEs en ovinos y caprinos. Asimismo, discutiremos su aplicabilidad y brechas en el conocimiento que puedan representar futuras áreas de estudio.

Se incluyen manuscritos sometidos a una revisión por pares y publicados durante las dos últimas décadas (2001-2021). Los métodos de inclusión de trabajos fueron establecidos bajo los siguientes criterios: (i) manuscritos que utilicen el enfoque in vivo, (ii) manuscritos realizados en ovinos y/o caprinos, (iii) manuscritos en los que haya presencia de una infección con NGI, (iv) manuscritos en los que se utilicen plantas ricas en terpenos (PRT) o AcEs y, (v) manuscritos en los que se relacione el consumo de dichas plantas o AcEs con la infección por NGI.

Tras el proceso de búsqueda y selección de manuscritos, se recuperaron 11 trabajos que cumplieron con los criterios de inclusión previamente establecidos. En la tabla 2 se observan algunos datos metodológicos generales. Brasil, con 7 trabajos es el país que lideró este tipo de investigaciones, seguido de 3 trabajos realizados en los Estados Unidos de América y uno en la república de Benín. En todos los manuscritos se reportó el uso del test de reducción del conteo fecal de huevos de NGI (TRCFH), mientras que 5 emplearon el test controlado (TCn). El $81 \%$ (9/11) de los manuscritos fue realizado en la especie ovina y solo el 19\% (2/11) utilizó caprinos. El promedio ( \pm desviación estándar) de animales utilizados fue de $27.6 \pm 12.3$. El $64 \%(7 / 11)$ de los manuscritos involucraron una infección natural por NGI, mientras que el $36 \%$ (3/11) optaron por una infección artificial, en su mayoría con el nematodo abomasal Haemonchus contortus $y$, en una ocasión, una infección mixta con $H$. contortus y el nematodo intestinal Trichostrongylus colubriformis.

La tabla 3 presenta los recursos y componentes químicos que estuvieron involucrados en los trabajos analizados. Aunque no se encontró una familia botánica sobre la que se dirijan esfuerzos específicos, tanto Lamiaceae $(n=2)$ como Rutaceae $(n=2)$ representaron el $36 \%$ de los trabajos (4/11). El uso de una planta con follaje rico en terpenos se dio en 2 de los 11 trabajos $(18 \%)$. Consecuentemente, la administración de AcEs ocupó la mayoría de los trabajos. En conjunto se documentaron 76 MSP obtenidos de los AcEs, dentro de los que se encontraron monoterpenos, sesquiterpenos, fenilpropanoides, hidrocarbones, ácidos, alcoholes, aldehídos, fenoles, ésteres acíclicos y lactonas. 
Torres-Fajardo y Higuera-Piedrahita - Control de nematodos gastrointestinales con terpenos y aceites esenciales

Tabla 2. Información general de manuscritos dirigidos a evaluar la actividad antihelmíntica de terpenos y aceites esenciales mediante experimentos in vivo en pequeños rumiantes.

\begin{tabular}{|c|c|c|c|c|c|c|c|c|}
\hline País & $\begin{array}{l}\text { Tipo de } \\
\text { trabajo }\end{array}$ & Especie & Raza & $\mathbf{n}$ & Edad & $\begin{array}{c}\text { Condición } \\
\text { experimental }\end{array}$ & $\begin{array}{c}\text { Tipo de } \\
\text { Infección }\end{array}$ & Referencia \\
\hline EUA & $\begin{array}{c}\text { TRCFH } \\
\text { TCn } \\
\text { TCn }\end{array}$ & Caprino & $\begin{array}{c}\text { Boer, Alpino } \\
\text { y Nubio }\end{array}$ & $\begin{array}{l}19 \\
30 \\
15\end{array}$ & $\begin{array}{c}0-5 \mathrm{a} \\
1-3.5 \mathrm{~m} \\
4-5 \mathrm{~m}\end{array}$ & Corral & $\begin{array}{l}\text { Natural } \\
\mathrm{ME}, \mathrm{Hc} \\
\mathrm{ME}, \mathrm{Hc}\end{array}$ & (62) \\
\hline Brasil & $\begin{array}{l}\text { TRCFH } \\
\text { TCn }\end{array}$ & Ovino & De Pelo & $\begin{array}{l}44 \\
21\end{array}$ & $\mathrm{NE}$ & Corral & Natural & (63) \\
\hline Brasil & TRCFH & Caprino & $\mathrm{NE}$ & 30 & $12-16 \mathrm{~m}$ & Campo & $\begin{array}{l}\text { Natural + } \\
\text { ME, Hc }\end{array}$ & (64) \\
\hline EUA & TRCFH & Ovino & Cruzas & 18 & $3 \mathrm{~m}$ & Corral & $\mathrm{ME}, \mathrm{Hc}$ & (65) \\
\hline Brasil & $\begin{array}{l}\text { TRCFH } \\
\text { TCn }\end{array}$ & Ovino & Santa Inés & 18 & $2 \mathrm{~m}$ & Corral & $\mathrm{ME}, \mathrm{HC}$ & (66) \\
\hline EUA & TRCFH & Ovino & $\begin{array}{l}\text { Blackbelly, } \\
\text { Saint Croix }\end{array}$ & 64 & $6 \mathrm{~m}$ & Corral & Natural & (67) \\
\hline Brasil & TRCFH & Ovino & $\mathrm{NE}$ & 30 & 6-16 m & Corral & Natural & (68) \\
\hline Brasil & TRCFH & Ovino & Santa Inés & 30 & $4-6 m$ & Corral & $\mathrm{ME}, \mathrm{HC}$ & (69) \\
\hline Benín & $\begin{array}{l}\text { TCn } \\
\text { TCn }\end{array}$ & Ovino & Djallonke & $\begin{array}{l}15 \\
15\end{array}$ & $4-6 m$ & $\begin{array}{l}\text { Campo } \\
\text { Corral }\end{array}$ & $\begin{array}{c}\text { Natural } \\
\text { Mix, Hc + Tc }\end{array}$ & (70) \\
\hline Brasil & TRCFH & Ovino & Santa Inés & 28 & $7 \mathrm{~m}$ & Corral & Natural & (71) \\
\hline Brasil & $\begin{array}{c}\text { TRCFH } \\
\text { TCn }\end{array}$ & Ovino & $\begin{array}{c}\text { Santa Inés, } \\
\text { Morada Nova }\end{array}$ & $\begin{array}{l}32 \\
34 \\
\end{array}$ & $\begin{array}{l}5 \mathrm{~m} \\
3 \mathrm{~m} \\
\end{array}$ & Corral & $\begin{array}{l}\mathrm{ME}, \mathrm{HC} \\
\text { Natural }\end{array}$ & (72) \\
\hline
\end{tabular}

País: EUA Estados Unidos de América; Tipo de trabajo: TRCFH Test de reducción del conteo fecal de huevos, TCn Test controlado; n número de animales experimentales; Edad: a años, m meses; Tipo de infección: ME Monoespecífica, HC Haemonchus contortus, Tc Trichostrongylus colubriformis

Tabla 3. Recursos utilizados, modo de administración y composición química de las plantas y/o aceites esenciales utilizados en los manuscritos analizados.

\begin{tabular}{|c|c|c|c|c|c|}
\hline Material & $\begin{array}{l}\text { Nombre } \\
\text { común }\end{array}$ & Familia & Presentación & Componentes químicos & Referencia \\
\hline $\begin{array}{l}\text { Chenopodium } \\
\text { ambrosioides }\end{array}$ & $\begin{array}{l}\text { Epazote, } \\
\text { paico }\end{array}$ & Amaranthaceae & $\begin{array}{c}\text { Follaje, Aceite } \\
\text { esencial }\end{array}$ & $\begin{array}{c}\text { [4]:Ascaridol, p-cimeno, a-terpineno, } \\
\text { limoneno }\end{array}$ & $(62)$ \\
\hline Lippia sidoides & $\begin{array}{l}\text { Pimienta de } \\
\text { romero }\end{array}$ & Verbenaceae & Aceite esencial & NE & $(63)$ \\
\hline $\begin{array}{l}\text { Eucalyptus } \\
\text { staigeriana }\end{array}$ & Eucalipto & Myrtaceae & Aceite esencial & $\begin{array}{c}\text { [20]: a-pineno, } \beta \text {-pineno, } \beta \text {-mirceno, } \\
\text { a-felandreno, } O \text {-cimeno, (+)- limoneno, } \\
\text { eucaliptol, y-terpineno, a-terpinoleno, } \\
\beta \text {-linalool, } \beta \text {-citronelal, } 4 \text {-terpineol, } \\
\text { a-terpineol, cis-geraniol, Z-citral, trans- } \\
\text { geraniol, E-citral, metil geranato, acetato de } \\
\text { nerol y acetato de geraniol }\end{array}$ & (64) \\
\hline Citrus sinensis & $\begin{array}{c}\text { Naranja } \\
\text { valenciana }\end{array}$ & Rutaceae & Aceite esencial & $d$-limoneno representó el $95 \%$ de la solución ${ }^{1}$ & $(65)$ \\
\hline $\begin{array}{l}\text { Cymbopogon } \\
\text { schoenantus }\end{array}$ & $\begin{array}{l}\text { Zacate } \\
\text { limón, } \\
\text { limoncillo }\end{array}$ & Poaceae & Aceite esencial & $\begin{array}{l}\text { [19]: Geraniol, geranial, neral, acetado de } \\
\text { geranil, citronelol, (E) cariofileno, linalool, } \\
\text { citronelal, (E)-2-hexenal, Y-cadineno, } \\
\text { óxido de cariofileno, N-decanal, 6-Metil-5- } \\
\text { hepteno-2-one, eugenol, acetato de citronelil, } \\
\text { a-humuleno, cadina 1,4 dieno, formato de } \\
\text { geranyl, a-muroleno }\end{array}$ & $(66)$ \\
\hline $\begin{array}{l}\text { Juniperus } \\
\text { pinchotii }\end{array}$ & $\begin{array}{l}\text { Enebro de } \\
\text { mora roja }\end{array}$ & Cupressaceae & Follaje & $\begin{array}{l}\text { La planta contiene buenas concentraciones de } \\
\text { taninos condensados y terpenoides }\end{array}$ & $(67)$ \\
\hline Thymus vulgaris & Tomillo & Lamiaceae & Aceite esencial & $\begin{array}{c}\text { [13]: Timol, p-cimeno, } \gamma \text {-terpineno, linalool, } \\
\beta \text {-duprezianeno, a-pineno, canfor, cis-hidrato } \\
\text { de sabineno, isoborneol, trans- } \beta \text {-terpineol, } \\
\text { mirceno, a-fencheno, }(Z) \text {-tagetona }\end{array}$ & (69) \\
\hline $\begin{array}{l}\text { Zanthoxylum } \\
\text { zanthoxyloïdes }\end{array}$ & Fagara & Rutaceae & Aceite esencial &  & $(70)$ \\
\hline Mentha arvensis & $\begin{array}{c}\text { Menta } \\
\text { Japonesa }\end{array}$ & Lamiaceae & Aceite esencial & $\begin{array}{c}\text { Mentol representó el } 86.7 \% \text { del aceite } \\
\text { esencial }{ }^{3}\end{array}$ & $(71)$ \\
\hline
\end{tabular}

NE: No especifica; ${ }^{1}$ Se administró una emulsión con el $40 \%$ del aceite de naranja, Componentes químicos: El número entre paréntesis representa la cantidad de metabolitos secundarios encontrados en el aceite esencial, 2 Reportan 25 metabolitos secundarios, pero solo se especifican los dispuestos en la tabla, ${ }^{3}$ No reportan más metabolitos secundarios. (68) y (72) no fueron incluidos puesto que utilizaron soluciones comerciales de monoterpenos aislados de aceites esenciales 
Torres-Fajardo y Higuera-Piedrahita - Control de nematodos gastrointestinales con terpenos y aceites esenciales

Los resultados que relacionan el consumo de terpenos y/o AcEs con la infección por NGI son presentados en la tabla 4. De manera general, la actividad $\mathrm{AH}$ de los terpenos o los AcEs fue variable, encontrando descensos porcentuales que oscilaron entre cero hasta el $97.4 \%$ de acuerdo con el TRCFH. El enfoque del TCn fue utilizado en 5 de los 11 trabajos (45\%) y al igual que el TRCFH, mostró resultados variables.

Tabla 4. Efecto bioactivo de plantas y aceites esenciales sobre nematodos gastrointestinales de pequeños rumiantes mediante la realización de pruebas in vivo.

\begin{tabular}{|c|c|c|c|c|c|c|}
\hline $\begin{array}{l}\text { Tipo de } \\
\text { trabajo }\end{array}$ & Material & Presentación & Dosis & $\begin{array}{c}\text { Resultados } \\
\text { TRCFH ( } \downarrow \text { hpg) }\end{array}$ & Resultados TCn & Referencia \\
\hline $\begin{array}{l}\text { TRCFH } \\
\text { TCn } \\
\text { TCn }\end{array}$ & $\begin{array}{l}\text { Chenopodium } \\
\text { ambrosioides }\end{array}$ & $\begin{array}{c}\text { Follaje, Aceite } \\
\text { esencial }\end{array}$ & $\begin{array}{l}0.2 \mathrm{~mL} / \mathrm{Kg} \\
250 \mathrm{~g} \text { hojas } \\
\mathrm{NE}\end{array}$ & NS & NS & $(62)$ \\
\hline $\begin{array}{l}\text { TRCFH } \\
\text { TCn }\end{array}$ & Lippia sidoides & Aceite esencial & $\begin{array}{c}230-283 \\
\mathrm{mg} / \mathrm{kg}\end{array}$ & $22.9-54.22 \%$ & $\begin{array}{l}\text { Hc representó el } 37.9 \% \text { de } \\
\text { la infección mientras que Tc } \\
\text { representó el } 62.1 \%\end{array}$ & $(63)$ \\
\hline TRCFH & $\begin{array}{l}\text { Eucalyptus } \\
\text { staigeriana }\end{array}$ & Aceite esencial & $350 \mathrm{mg} / \mathrm{kg}$ & $73.66 \%$ & NA & (64) \\
\hline TRCFH & Citrus sinensis & Aceite esencial & $600 \mathrm{mg} / \mathrm{kg}^{1}$ & $94.9-97.4 \%$ & NA & $(65)$ \\
\hline $\begin{array}{l}\text { TRCFH } \\
\text { TCn }\end{array}$ & $\begin{array}{l}\text { Cymbopogon } \\
\text { schoenantus }\end{array}$ & Aceite esencial & $180-360 \mathrm{mg} / \mathrm{kg}$ & NS & NS & $(66)$ \\
\hline TRCFH & $\begin{array}{c}\text { Juniperus } \\
\text { pinchotii }\end{array}$ & Follaje & Inclusión $30 \%$ & $65 \%$ & NA & (67) \\
\hline TRCFH & $\begin{array}{c}\text { Acetato de } \\
\text { carvacryl }\end{array}$ & Aceite esencial & $250 \mathrm{mg} / \mathrm{kg}$ & $65.9 \%$ & NA & $(68)$ \\
\hline TRCFH & Thymus vulgaris & Aceite esencial & $\begin{array}{c}75-150-300 \\
\mathrm{mg} / \mathrm{kg}\end{array}$ & NS & NA & (69) \\
\hline $\begin{array}{l}\text { TCn } \\
\text { TCn }\end{array}$ & $\begin{array}{l}\text { Zanthoxylum } \\
\text { zanthoxyloides }\end{array}$ & Aceite esencial & $1-2 \mathrm{~mL} / \mathrm{Kg}^{2}$ & $89 \%$ & $\begin{array}{l}\text { El número de NGI adultos se } \\
\text { redujo. } \\
\text { La prolificidad de las hembras } \\
\text { también se vio reducida }\end{array}$ & (70) \\
\hline TRCFH & Mentha arvensis & Aceite esencial & $160-200 \mathrm{mg} / \mathrm{kg}$ & $\begin{array}{c}44.9-61.6 \% \\
(N S)\end{array}$ & NA & (71) \\
\hline $\begin{array}{l}\text { TRCFH } \\
\text { TCn }\end{array}$ & $\begin{array}{c}\text { Anetol y carvona } \\
\text { encapsulados }\end{array}$ & Aceite esencial & $100-250 \mathrm{mg} / \mathrm{kg}^{*}$ & $\begin{array}{c}55 \% \text { a los } 47 \\
\text { días }\end{array}$ & Se redujo la fertilidad de los NGI & $(72)$ \\
\hline
\end{tabular}

\section{El enfoque in vivo en la evaluación de compuestos con potencial bioactivo en pequeños rumiantes}

La finalidad de la presente revisión de literatura fue analizar algunos manuscritos científicos que evaluaron la actividad $\mathrm{AH}$ de terpenos y AcEs en pequeños rumiantes mediante la realización de pruebas in vivo. Este enfoque, en el que se involucra directamente el factor animal ha representado un componente imprescindible en la evaluación de productos naturales en las ciencias veterinarias. Sin embargo, es necesario recalcar que aún no existen guías oficiales para determinar la eficacia de productos naturales en rumiantes. Por tal razón, resulta necesario enfatizar en la necesidad de elaborar e implementar procedimientos avalados por instancias oficiales. La comunidad de parasitólogos veterinarios esperamos que en el futuro próximo entidades internacionales como la asociación mundial para el avance de la parasitología veterinaria o el organismo de cooperación internacional en la harmonización de requerimientos técnicos para el registro de productos médicos veterinarios (WAAVP y 
VICH por sus siglas en inglés, respectivamente) difundan dichos documentos.

En consecuencia, los trabajos realizados hasta la fecha han empleado las guías oficiales para determinar la efectividad de productos sintéticos (73) o algunas publicaciones realizadas por expertos en la materia (74). Este tipo de estrategias comprenden el estudio de las propiedades $\mathrm{AH}$ de plantas o sus extractos sobre diferentes etapas del ciclo biológico de los NGI (huevos, larvas y adultos) a nivel de laboratorio, constituyendo el denominado enfoque in vitro.

Las pruebas in vitro presentan un bajo costo, mayor rapidez en los resultados y otorgan la posibilidad de examinar un número relativamente amplio de materiales en un periodo corto de tiempo (75), por lo que han sido más utilizadas y documentadas. Basados en nuestra búsqueda de literatura, podemos afirmar que por cada siete a diez manuscritos que emplean el enfoque in vitro, solo uno utiliza el enfoque in vivo. Sin embargo, las pruebas in vitro no se enfrentan a la naturaleza multifactorial de la fisiología animal en la cual: (i) existe una dinámica en las cantidades y concentraciones de químicos que ingresan al sistema digestivo, (ii) la tasa y secuencia en la que los MSP ingresan y son procesados es variable y dinámica, (iii) se requeriría de consumos elevados de material vegetal fresco por parte de los animales para igualar las concentraciones que arrojaron resultados positivos en condiciones in vitro $y$, (iv) las poblaciones microbianas del rumen tienen la capacidad de desarrollar adaptaciones a los MSP. Por lo tanto, debido a la dificultad de duplicar estos factores, evaluar en el huésped es la mejor alternativa para determinar el valor $\mathrm{AH}$ de plantas y sus derivados (75).

Para el desarrollo del enfoque in vivo en la evaluación de la actividad $\mathrm{AH}$ de productos naturales se pueden implementar dos metodologías (72):

Test de reducción del conteo fecal de huevos de NGI (TRCFH); en el que se administra el material bioactivo a un grupo de animales y se hace seguimiento cuantitativo de la excreción de huevos por gramo (HPG) de NGI en un periodo de tiempo (pre y pos) que debe ser establecido por el investigador. En este enfoque la presencia de un grupo control -en el cual los animales no reciben el material bioactivo o se encuentran libres de una infección por NGI- es opcional.
Test controlado (TCn); el cual presenta los mismos fundamentos del TRCFH, pero con la obligatoriedad de establecer un grupo control y de realizar sacrificios humanitarios a los animales experimentales con el objetivo de recuperar los órganos del tracto gastrointestinal donde se alojan los NGI adultos. Posteriormente se deben realizar conteos de las poblaciones de NGI al igual que mediciones en los NGI recuperados, lo que permite una comparación entre grupos experimentales. Esta metodología, a pesar de ser más costosa y necesitar mayor mano de obra, es la más confiable para evaluar la actividad $\mathrm{AH}$ de un producto natural.

\section{Experiencias en la utilización de terpenos $y$ aceites esenciales sobre los nematodos gastrointestinales de ovinos y caprinos}

El TRCFH fue utilizado en todos los trabajos incluidos en la presente revisión. Los resultados de la dinámica en las cuentas de HPG tras la administración de PRT o AcEs fueron variables, mostrando actividades que pueden clasificarse como nulas $(62,66,69)$, moderadas $(63,67,68)$, positivas $(64,70)$ y sobresalientes $(65)$. Si bien estas respuestas en la dinámica de la infección por NGI provienen de un número reducido de trabajos y son ciertamente ambiguas, nos permiten encontrar un potencial en la administración de estos recursos con fines terapéuticos en pequeños rumiantes. Futuras investigaciones podrían realizarse considerando factores que pueden influenciar las propiedades bioactivas de los recursos naturales como la dosis, biodisponibilidad, época de recolecta de las especies de plantas, método de extracción de MSP o AcEs, presencia de otros MSP e interacciones entre MSP. De igual manera se deben considerar factores relacionados con los animales como la vía de administración, comportamiento productivo, plano nutricional, estado fisiológico y nivel de adaptación a MSP.

En la presente revisión, cinco estudios implementaron el enfoque del TCn para la evaluación de la actividad AH de PRT o AcEs y, de manera similar con el TRCFH los resultados fueron variables. En los trabajos de Ketzis et al (62) y Katiki et al (66), la administración del AcEs de Chenopodium ambrosioides y Cymbopogon schoenantun respectivamente, no tuvo efecto sobre el número de NGI adultos recuperados en la necropsia. Resultados significativos fueron reportados por Camurca et al (61) quienes mencionan cierto nivel de selectividad del AcEs 
de Lippia sidoides sobre las poblaciones de Haemonchus contortus. Asimismo, el AcEs de los frutos de Zanthoxylum zanthoxyloides (70) y una solución encapsulada de los monoterpenos anetol y carvona (72) disminuyeron el número de NGI adultos, el tamaño de los machos y el número de huevos producidos por hembra.

Los mecanismos de acción por los que los terpenos o AcEs afectan a los NGI aún son inciertos. Sin embargo, se ha propuesto que, debido a su naturaleza hidrofóbica, la membrana celular es el objetivo primario (76). Estos compuestos ingresan a las estructuras de los NGI a través de difusión transcuticular, interfiriendo con el metabolismo, impidiendo funciones vitales desde etapas tempranas de desarrollo y afectando algunos mecanismos de locomoción (77). De acuerdo con López y Pascual-Villalobos (78), los AcEs presentan actividad hacia los receptores de acetilcolinesterasa presentes en vertebrados e invertebrados produciendo un daño neurotóxico similar a los organofosforados. Sin embargo, resulta importante recalcar que la bioactividad de terpenos y AcEs sobre otros organismos puede ser tan amplia y diversa como su elevado número de moléculas identificadas.

La investigación y el desarrollo de productos naturales con propiedades $\mathrm{AH}$ ha surgido como una respuesta hacia las crecientes poblaciones de NGI resistentes a los AH comerciales. Durante esta revisión, la existencia de poblaciones de NGI resistentes a la ivermectina se hizo evidente, al reportar reducciones en la eliminación de HPG del orden de $67-85 \%$ (63), 35 - 54\% (62) y $25 \%$ (67). Asimismo, algunos estudios utilizaron un medicamento $\mathrm{AH}$ de reciente introducción en el mercado (Zolvix $囚$ ) encontrando niveles de reducción en la eliminación de HPG de $100 \%$ (69), 96.4\% (68) y, aunque los gráficos presentados por Katiki et al (72) carecen de un dato específico, se puede observar -a reserva de confirmar- cierto nivel de resistencia hacia este medicamento después de 21 días de ser aplicado. El estudio de estas variables parasitológicas se puede ver complementado con el seguimiento de variables fisiológicas como el volumen del paquete celular (o hematocrito), FAMACHA® y recuento de células blancas $(66,67,70,72)$, junto con la evaluación de la función renal y hepática $(66,72)$. Adicionalmente, el seguimiento de variables productivas como la ganancia diaria de peso (GDP) y la conversión alimenticia reviste una gran importancia en términos de aplicabilidad en diferentes sistemas productivos. En este sentido, Whitney et al (67) reportan una disminución en la ganancia de peso tras la inclusión del 30\% de Juniperus pinchotii en una dieta balanceada en corderos Blackbelly y Saint Croix. Estos resultados son similares a los reportados por Katiki et al (72) quienes reportan una disminución en la GDP en corderos Santa Inés. Por su parte, Azando et al (70) reportan que la administración del AcEs de los frutos de Z. zanthoxylum no afectó la GDP ni el peso vivo final de corderos Djallonke. En este sentido, a pesar de que los resultados tienden a indicar una afectación en las variables productivas, futuros trabajos deben establecer los costos del trade-off entre nutrición y sanidad (79-80).

Los terpenos y AcEs poseen un amplio espectro de actividades biológicas que pueden ser exploradas en las ciencias veterinarias. Recientemente se reportó que el $58 \%$ de 113 manuscritos que hicieron parte de una revisión global sobre terpenos en animales, estuvieron dirigidos a aquellos destinados para consumo humano (81). Actualmente se han identificado unas 80,000 plantas que ejercen alguna influencia sobre la salud y la productividad animal (82), por lo tanto, resulta necesario conocer la naturaleza de sus interacciones con los herbívoros y de esta forma poder sugerir su uso como recursos que representen un beneficio nutricional y farmacéutico en los sistemas de producción de ovinos y caprinos $(83,84)$. Estos beneficios podrían estar representados en los denominados alimentos funcionales o recursos nutracéuticos.

\section{Conflicto de intereses}

Los autores del presente estudio declaramos que no existe conflicto de intereses con la publicación de este manuscrito. 


\section{REFERENCIAS}

1. Mavrot $F$, Hertzberg $H$, Torgerson P. Effect of gastro-intestinal nematode infection on sheep performance: A systematic review and meta-analysis. Parasit Vectors. 2015; 8(1):1-11. http://dx.doi.org/10.1186/ $\underline{\text { s13071-015-1164-Z }}$

2. Zajac AM, Garza J. Biology, Epidemiology, and Control of Gastrointestinal Nematodes of Small Ruminants. Vet Clin North Am - Food Anim Pract. 2020; 36(1):73-87. https://doi.org/10.1016/j.cvfa.2019.12.005

3. Kaplan RM, Vidyashankar AN. An inconvenient truth: global worming and anthelmintic resistance. Vet Parasitol. 2012; 186(1-2):70-78. https://doi.org/10.1016/j. vetpar.2011.11.048

4. Torres-Acosta JFJ, Mendoza-de-Gives $P$, Aguilar-Caballero AJ, Cuéllar-Ordaz JA. Anthelmintic resistance in sheep farms: update of the situation in the American continent. Vet Parasitol. 2012; 189(1):89-96. https://doi.org/10.1016/j. vetpar.2012.03.037

5. Scott I, Pomroy WE, Kenyon PR, Smith G, Adlington B, Moss A. Lack of efficacy of monepantel against Teladorsagia circumcinta and Trichostrongylus colubriformis. Vet Parasitol. 2013; 198(1-2):166-171. https:// doi.org/10.1016/j.vetpar.2013.07.037

6. Van-de-Brom R, Moll L, Kappert C, Vellema P. Haemonchus contortus resistance to monepantel in sheep. Vet Parasitol; 2015; 209(3-4):278-280. https://doi. org/10.1016/j.vetpar.2017.09.010

7. Salles N, Love S. Resistance of Haemonchus sp. to monepantel and reduced efficacy of a derquantel / abamectin combination confirmed in sheep in NSW, Australia. Vet Parasitol. 2016; 228:193-196. https://doi. org/10.1016/j.vetpar.2016.08.016

8. Cerutti J, Cooper L, Torrents J, Suárez G, Anziani OS. Eficacia reducida de derquantel y abamectina en ovinos y caprinos con Haemonchus sp resistentes a lactonas macrocíclicas. Rev Vet. 2018; 29(1):22-25. http://dx.doi.org/10.30972/vet.2912782
9. Charlier J, Rinaldi L, Musella V, Ploeger $\mathrm{HW}$, Chartier $\mathrm{C}$, Rose Vineer $\mathrm{H}$, et al. Initial assessment of the burden of parasitic helminth infections to the ruminant livestock industry in Europe. Prev Vet Med. 2020; 182:105103. https://doi.org/10.1016/j. prevetmed.2020.105103

10. Charlier J, van der Voort M, Kenyon F, Skuce $P$, Vercruysse $\mathrm{J}$. Chasing helminths and their economic impact on farmed ruminants. Trends Parasitol. 2014; 30(7):361-367. https://doi.org/10.1016/j.pt.2014.04.009

11. Torres-Acosta JFJ, Hoste H, Sandoval-Castro CA, Torres-Fajardo RA, Ventura-Cordero J, González-Pech PG, et al. The art of war against gastrointestinal nematodes in sheep and goat herds of the tropics. Rev Acad (Pontif Univ Catól Paraná, Online). 2019; 17(1):39-46. https://periodicos.pucpr.br/ index.php/cienciaanimal/issue/view/1977

12. Burke JM, Miller JE. Sustainable approaches to parasite control in ruminant livestock. Vet Clin North Am Food Anim Pract. 2020; 36(1):89-107. https://doi.org/10.1016/j. cvfa.2019.11.007

13. Torres-Acosta JFJ, Sandoval-Castro CA, Hoste $\mathrm{H}$, Aguilar-Caballero AJ, CámaraSarmiento MA, Alonso-Díaz MA. Nutritional manipulation of sheep and goats for the control of gastrointestinal nematodes under hot humid and subhumid tropical conditions. Small Rum Res. 2012; 103(1):28-40. https://doi.org/10.1016/j. smallrumres.2011.10.016

14. Hoste H, Torres-Acosta JFJ, Quijada J, Chan-Pérez I, Dakheel MM, Kommuru DS, et al. Interactions between nutrition and infections with Haemonchus contortus and related gastrointestinal nematodes in small ruminants. Adv Parasitol. 2016; 93:239-351. https://doi.org/10.1016/ bs.apar.2016.02.025

15. Heckendorn F, Bieber A, Werne A, Saratsis A, Maurer V, Stricker C. The genetic basis for the selection of dairy goats with enhanced resistance to gastrointestinal nematodes. Parasite. 2017; 24:32. https:// doi.org10.1051/parasite/2017033

16. Bishop SC. A consideration of resistance and tolerance for ruminant nematode infections. Front Genet. 2012; 3:168. https://doi. org/10.3389/fgene.2012.00168 
Torres-Fajardo y Higuera-Piedrahita - Control de nematodos gastrointestinales con terpenos y aceites esenciales

17. Claerebout $E$, Geldholf P. Helminth vaccines in ruminants: From development to application. Vet Clin North Am Food Anim Pract. 2020; 36(1):159-171. https://doi. org/10.1016/j.cvfa.2019.10.001

18. Ehsan $M$, Hu RS, Liang $Q L$, Hou JL, Song $X$, Yan $\mathrm{R}$, et al. Advances in the development of anti-Haemonchus contortus vaccines: Challenges, opportunities and perspectives. Vaccines. 2020; 8(3):555. https://doi. org/10.3390/vaccines8030555

19. Galindo-Barboza AJ, Torres-Acosta JFJ, Cámara-Sarmiento R, Sandoval-Castro CA, Aguilar-Caballero AJ, Ojeda-Robertos NF, et al. Persistence of the efficacy of copper oxide wire particles against Haemonchus contortus in sheep. Vet Parasitol. 2011; 176(23): 201-207. https://doi.org/10.1016/j. vetpar.2010.11.012

20. Whitley NC, Dykes G, Vazquez J, Burke JM, Terrill T. Effect of Copper Oxide Wire Particles without anthelmintic treatment or anthelmintic treatment alone on gastrointestinal nematode (GIN) fecal egg counts in goats. J Anim Sci; 2021: 99(Suppl. S2). https://doi-org.ezproxy.javeriana.edu. co/10.1093/jas/skab096.079

21. Mahieu M, Arquet R, Fleury J, Bonneau $M$, Mandonnet $N$. Mixed grazing of adult goats and cattle: Lessons from longterm monitoring. Vet Parasitol. 2020; 280:109087. https://doi.org/10.1016/j. vetpar.2020.109087

22. Szewc M, De Waal T, Zintl A. Biological methods for the control of gastrointestinal nematodes. Vet J. 2021; 268:105602. https://doi.org/10.1016/j.tvjl.2020.105602

23. Comans-Pérez RJ, Sánchez JE, Al-Ani LKT, González-Cortázar M, Castañeda-Ramírez GS, Mendoza-de-Gives P, et al. Biological control of sheep nematode Haemonchus contortus using edible mushrooms. Biol Control. 2021; 152:104420. https://doi. org/10.1016/j.biocontrol.2020.104420

24. Borges DGL, Borges FA. Plants and their medicinal potential for controlling gastrointestinal nematodes in ruminants. Nematoda. 2016; 3e:92016. https://dx.doi. org/10.4322/nematoda.00916

25. García-Bustos JF, Sleebs BE, Gasser RB. An appraisal of natural products active against parasitic nematodes of animals. Parasit Vectors. 2019; 12(1):1-22. https://doi. org/10.1186/s13071-019-3537-1
26. Liu M, Panda SK, Luyten W. Plant-based natural products for the discovery and development of novel anthelmintics against nematodes. Biomolecules. 2020; 10(3):426. https://doi.org/10.3390/biom 10030426

27. Mithöfer A, Boland W. Plant defense against herbivores: Chemical aspects. Annu Rev Plant Biol. 2012; 63:431450. https://doi.org/10.1146/annurevarplant-042110-103854

28. Agrawal AA, Weber MG. On the study of plant defence and herbivory using comparative approaches: how important are secondary plant compounds. Ecol Lett. 2015; 18(10):985-991. https://doi. org/10.1111/ele.12482

29. Ma T, Gao H, Zhang D, Shi Y, Zhang T, Shen $X$, Wu S, Xiang L, Chen S. Transcriptome analyses revealed the ultraviolet $B$ irradiation and phytohormone gibberellins coordinately promoted the accumulation of artemisinin in Artemisia annua L. Chin Med. 2020; 15:67. https://doi.org/10.1186/s13020$\underline{020-00344-8}$

30. De Morais LAS. Influência dos fatores abióticos na composição química dos óleos essenciais. Hortic Bras. 2009; 27(2):S4050-4063. https://ainfo.cnptia.embrapa.br/digital/ bitstream/item/143457/1/2009AA-051.pdf

31. Yang L, Wen KS, Ruan X, Zhao YX, Wei F, Wang Q. Response of plant secondary metabolites to environmental factors. Molecules. 2028; 23(4):762. https://doi. org/ $10.3390 /$ molecules 23040762

32. Neilson EH, Goodger JQD, Woodrow IE, Møller BL. Plant chemical defense: At what cost?. Trends Plant Sci. 2013; 18(5):250258. http://dx.doi.org/10.1016/j. tplants.2013.01.001

33. Mueller-Harvey I, Bee G, Dohme-Meier F, Hoste $\mathrm{H}$, Karonen M, Kölliker R, et al. Benefits of condensed tannins in forage legumes fed to ruminants: Importance of structure, concentration, and diet composition. Crop Sci. 2019; 59(3):861-885. https://doi. org/10.2135/cropsci2017.06.0369

34. Villalba JJ, Costes-Thiré M, Ginane C. Phytochemicals in animal health: Diet selection and trade-offs between costs and benefits. Proc Nutr Soc. 2017; 76(2):113-121. https://doi.org/10.1017/ $\underline{\text { S0029665116000719 }}$ 
35. Muir J. The multi-faceted role of condensed tannins in the goat ecosystem. Small Rumin Res. 2011; 98(1-3):115-120. http://dx.doi. org/10.1016/j.smallrumres.2011.03.028

36. Hackmann TJ, Spain JN. Invited review: Ruminant ecology and evolution: Perspectives useful to ruminant livestock research and production. J Dairy Sci. 2010; 93(4):1320-1334. https://doi.org/10.3168/ jds.2009-2071

37. Wang S, Alseekh S, Fernie AR, Luo J. The Structure and Function of Major Plant Metabolite Modifications. Mol Plant. 2019; 12(7):899-919. https://doi.org/10.1016/j. molp.2019.06.001

38. Pichersky E, Lewinsohn E. Convergent evolution in plant specialized metabolism. Annu Rev Plant Biol. 2011; 62:4966. https://doi.org/10.1146/annurevarplant-042110-103814

39. Staniek A, Bouwmeester $H$, Fraser PD, Kayser O, Martens S, Tissier A, et al. Natural products - modifying metabolite pathways in plants. Biotechnol J. 2013; 8(10):1159-71. https://doi.org/10.1002/biot.201300224

40. Dubois $\mathrm{O}$, Allanic $\mathrm{C}$, Charvet $\mathrm{CL}$, Guégnard $F$, Février $H$, Théry-Koné $I$, et al. Lupin (Lupinus spp.) seeds exert anthelmintic activity associated with their alkaloid content. Sci Rep. 2019. 9(1):1-12. https:// doi.org/10.1038/s41598-019-45654-6

41. Herath HMPD, Preston S, Jabbar A, García-Bustos J, Taki AC, Addison RS, et al. Identification of Fromiamycalin and Halaminol A from Australian marine sponge extracts with anthelmintic activity against Haemonchus contortus. Mar Drugs. 2019; 17:598. https://doi.org/10.3390/ $\underline{\mathrm{md} 17110598}$

42. Spiegler V, Liebau E, Hensel A. Medicinal plant extracts and plant-derived polyphenols with anthelmintic activity against intestinal nematodes. Nat Prod Rep. 2017; 34(6):627643. https://doi.org/10.1039/c6np00126b

43. Oliveira Santos F, Ponce Morais Cerqueira A, Branco A, José Moreira Batatinha $M$, Borges Botura M. Anthelmintic activity of plants against gastrointestinal nematodes of goats: A review. Parasitology. 2019;146(10):1233-1246. https://doi. org/10.1017/S0031182019000672
44. Hoste H, Martínez-Ortíz-de-Montellano C, Manoralaki F, Brunet S, Ojeda-Robertos $\mathrm{N}$, Fourquaux I, et al. Direct and indirect effect of bioactive tannin-rich tropical and temperate legumes against nematode infections. Vet Parasitol. 2012; 186(12):18-27. https://doi.org/10.1016/j. vetpar.2011.11.042

45. Piluzza G, Sulas L, Bullita S. Tannins in forage plants and their role in animal husbandry and environmental sustainability: A review. Grass Forage Sci. 2014; 69(1):32-48. https://doi.org/10.1111/gfs.12053

46. Zhou F, Pichersky E. More is better: the diversity of terpene metabolism in plants. Curr Opin Plant Biol. 2020; 55:1-10. https:// doi.org/10.1016/j.pbi.2020.01.005

47. Bodas R, Prieto N, García-González R, Andrés S, Giráldez FJ, López S. Manipulation of rumen fermentation and methane production with plant secondary metabolites. Anim Feed Sci Technol. 2012; 176(1-4):78-93. https://doi. org/10.1016/j.anifeedsci.2012.07.010

48. Gershenzon J, Dudareva N. The function of terpene natural products in the natural world. Nat Chem Biol. 2007; 3:408-414. https://doi.org/10.1038/nchembio.2007.5

49. Mudianta IW, White AM, Suciati, Katavic $P L$, Krishnaraj RR, Winters $A E$, et al. Chemoecological studies on marine natural products: Terpene chemistry from marine mollusks. Pure Appl Chem. 2014; 86(6): 995-1002. https://doi.org/10.1515/ pac-2013-1111

50. Dudareva N, Negre F, Nagegowda DA, Orlova I. Plant volatiles: recent advances and future perspectives. Crit Rev Plant Sci. 2006; 25:417-440. https://doi. org/10.1080/07352680600899973

51. Bruce TJA, Pickett JA. Perception of plant volatile blends by herbivorous insects Finding the right mix. Phytochemistry. 2011; 72(13):1605-1611. https://doi. org/10.1016/j.phytochem.2011.04.011

52. Benchaar C, Calsamiglia S, Chaves AV, Fraser GR, Colombatto D, McAllister TA, Beauchemin KA. A review of plant-derived essential oils in ruminant nutrition and production. Anim Feed Sci Technol. 2008; 145:209-228. https://doi.org/10.1016/j. anifeedsci.2007.04.014 
53. García C, Montero G, Coronado MA, Valdez B, Stoytcheva M, Rosas N, et al. Valorization of Eucalyptus Leaves by Essential Oil Extraction as an Added Value Product in Mexico. Waste and Biomass Valorization. 2017;8(4):11871197. https://doi.org/10.1007/s12649$\underline{016-9695-x}$

54. Torres RNS, Moura DC, Ghedini CP, Ezequiel JMB, Almeida MTC. Meta-analysis of the effects of essential oils on ruminal fermentation and performance of sheep. Small Rumin Res. 2020; 189:106148. https://doi. org/10.1016/j.smallrumres.2020.106148

55. Cobellis G, Trabalza-Marinucci M, Yu Z. Critical evaluation of essential oils as rumen modifiers in ruminant nutrition: A review. Sci Total Environ. 2016; 545546:556-568. http://dx.doi.org/10.1016/j. scitotenv.2015.12.103

56. Pavela R. Essential oils for the development of eco-friendly mosquito larvicides: A review. Ind Crops Prod. 2015; 76:174-187. http:// dx.doi.org/10.1016/j.indcrop.2015.06.050

57. Bhavaniramya $S$, Vishnupriya $S, A l-A b o o d y$ MS, Vijayakumar R, Baskaran D. Role of essential oils in food safety: Antimicrobial and antioxidant applications. Grain Oil Sci Technol. 2019; 2(2):49-55. https://doi. org/10.1016/j.gaost.2019.03.001

58. Srivastava A, Lall R, Sinha A, Gupta RC. Essential Oils. En Nutraceuticals in Veterinary Medicine. Gupta R, Srivastava A, Lall R (Eds.). Switzerland: Springer Nature; 2019. https:// doi.org/10.1007/978-3-030-04624-8

59. Mukherje N, Mukherjee S, Saini P, Roy P, Babu S. Phenolics and terpenoids; the promising new search for anthelmintics: A critical review. Mini-Reviews Med Chem. 2016; 16(17):1415-1441. https://doi.org/ 10.2174/1389557516666151120121036

60. Abdel-Rahman FH, Alaniz NM, Saleh MA. Nematicidal activity of terpenoids. J Environ Sci Heal B. 2013; 48(1):16-22. https://doi. org/10.1080/03601234.2012.716686

61. André WPP, Ribeiro WLC, Oliveira LMB, Macedo ITF, Rondon FCM, Bevilaqua CML. Óleos essenciais e seus compostos bioativos no controle de nematoides gastrintestinais de pequenos ruminantes. Acta Sci Vet. 2018; 46:1522. https://doi.org/10.22456/1679$\underline{9216.81804}$
62. Ketzis JK, Taylor A, Bowman DD, Brown DL, Warnick LD, Erb HN. Chenopodium ambrosioides and its essential oils as treatments for Haemonchus contortus and mixed adult-nematode infections in goats. Small Rum Res. 2002; 44(3):193200. https://doi.org/10.1016/S09214488(02)00047-0

63. Camurça-Vasconcelos ALF, Bevilaqua CML, Morais SM, Maciel MV, Costa CTC, Macedo ITF, et al. Anthelmintic activity of Lippia sidoides essential oil on sheep gastrointestinal nematodes. Vet Parasitol. 2008; 154(1-2):167-170. https://doi. org/10.1016/j.vetpar.2008.02.023

64. Macedo ITF, Bevilaqua CML, Oliveira LMB, Camurça-Vasconcelos ALF, Vieira LS, Oliveira FR, et al. Anthelmintic effect of Eucalyptus staigeriana essential oil against gastrointestinal nematodes. Vet Parasitol. 2010; 173(1-2):93-98. https:// doi.org/10.1016/j.vetpar.2010.06.004

65. Squires JM, Foster JG, Lindsay DS, Caudell DL, Zajac AM. Efficacy of an orange oil emulsion as an anthelmintic against Haemonchus contortus in gerbils (Meriones unguiculatus) and in sheep. Vet Parasitol. 2010; 172(1-2):95-99. https://doi. org/10.1016/j.vetpar.2010.04.017

66. Katiki LM, Chagas ACS, Takahira RK, Juliani HR, Ferreira JFS, Amarante AFT. Evaluation of Cymbopogon schoenanthus essential oil in lambs experimentally infected with Haemonchus contortus. Vet Parasitol. 2012; 186(3-4):312-318. https://doi. org/10.1016/j.vetpar.2011.12.003

67. Whitney TR, Wildeus S, Zajac AM. The use of redberry juniper (Juniperus pinchotii) to reduce Haemonchus contortus fecal egg counts and increase ivermectin efficacy. Vet Parasitol. 2013; 197(1-2):82-188. https:// doi.org/10.1016/j.vetpar.2013.06.010

68. Andre WPP, Ribeiro WLC, Cavalcante GS, Santos, JML, Macedo ITF, Paula HCB, et al. Comparative efficacy and toxic effects of carvacryl acetate and carvacrol on sheep gastrointestinal nematodes and mice. Vet Parasitol. 2016; 218:52-58. https://doi. org/10.1016/j.vetpar.2016.01.001

69. Ferreira LE, Benincasa BI, Fachin AL, França SC, Contini SSHT, Chagas ACS, Beleboni RO. Thymus vulgaris $\mathrm{L}$. essential oil and its main component thymol: Anthelmintic effects against Haemonchus contortus from sheep. Vet Parasitol. 2016; 288:7076. https://doi.org/10.1016/j.vetpar.2016.08.011 
70. Azando EVB, Olounlade AP, HounzangbeAdote MS, Tam Ha TB, Fabre N, Valentin A. Contrôle des parasitoses gastro-intestinales ovines par I'huile essentielle de Zanthoxylum zantoxyloïdes (Fagara zantoxyloïdes). Rev Med Vet. 2017; 168:205-212. https://www. revmedvet.com/2017/RMV168 205 212.pdf

71. Chagas ACS, Figuereido A, Politi FAS, Moro IJ, Esteves SN, Bizzo HR, Gama PE, Chaves FCM. Efficacy of essential oils from planta cultivated in the Amazonian Biome against gastrointestinal nematodes in sheep. J Parasit Dis. 2018; 42:357-364. https://doi. org/10.1007/s12639-018-1007-x

72. Katiki LM, Araujo RC, Ziegelmeyer L, Gomes ACP, Gutmanis G, Rodrigues L, et al. Evaluation of encapsulated anethole and carvone in lambs artificially- and naturally - infected with Haemonchus contortus. Exp Parasitol. 2019; 197:36-42. https://doi. org/10.1016/j.exppara.2019.01.002

73. Wood IB, Amaral NK, Bairden K, Duncan JL, Kassai T, Malone JB, et al. World Association for the Advancement of Veterinary Parasitology (W.A.A.V.P.) second edition of guidelines for evaluating the efficacy of anthelmintics in ruminants (bovine, ovine, caprine). Vet Parasitol. 1995; 58:181-213. https://doi. org/10.1016/0304-4017(95)00806-2

74. Jackson $F$, Hoste $H$. In vitro methods for the primary screening of plant products for direct activity against ruminant gastrointestinal nematodes. En In Vitro Screening of Plant Resources for Extra Nutritional Attributes in Ruminants: Nuclear and Related Methodologies; Vercoe PE, Makkar HPS, Schlink AC (Eds.). FAO/IAEA Springer Edition: Dordrecht, The Netherlands; 2010.

75. Villalba JJ, Provenza FD. Challenges in Extrapolating In vitro Findings to In Vivo Evaluation of Plant Resources. En In Vitro Screening of Plant Resources for Extra Nutritional Attributes in Ruminants: Nuclear and Related Methodologies; Vercoe PE, Makkar HPS, Schlink AC (Eds.). FAO/ IAEA Springer Edition: Dordrecht, The Netherlands; 2010.

76. Castilho CVV, Fantatto RR, Gaínza YA, Bizzo HR, Barbi NS, Leitão SG, et al. In vitro activity of the essential oil from Hesperozygis myrtoides on Rhipicephalus (Boophilus) microplus and Haemonchus contortus. Rev Bras Farmacogn. 2017; 27(1): 70-76. http://dx.doi.org/10.1016/j. bjp.2016.08.005
77. Katiki LM, Barbieri AME, Araujo RC, Veríssimo CJ, Louvandini $\mathrm{H}$, Ferreira JFS. Synergistic interaction of ten essential oils against Haemonchus contortus in vitro. Vet Parasitol. 2017; 243: 47-51. http://dx.doi. org/10.1016/j.vetpar.2017.06.008

78. López MD, Pascual-Villalobos MJ. Mode of inhibition of acetylcholinesterase by monoterpenoids and implications for pest control. Ind Crop Prod. 2010; 31:284-288. https://doi.org/10.1016/j. indcrop.2009.11.005

79. Costes-Thiré M, Laurent P, Ginane C, Villalba JJ. Diet selection and trade-offs between condensed tannins and nutrients in parasitized sheep. Vet Parasitol. 2019; 271:14-21. https://doi.org/10.1016/j. vetpar.2019.05.013

80. Landau SY, Provenza FD. Of browse, goats, and men: Contribution to the debate on animal traditions and cultures. Appl Anim Behav Sci. 2020; 232:105127. https://doi. org/10.1016/j.applanim.2020.105127

81. Da Silva JJM, Campanharo SC, Paschoal JAR. Ethnoveterinary for food-producing animals and related food safety issues: A comprehensive overview about terpenes. Compr Rev Sci Food Saf. 2021; 20(1):1-43 https://doi.org/10.1111/1541-4337.12673

82. Zeineldin $M M$, Sabek $A A$, Barakat RA, Elghandour MMMY, Salem AZ, Jiménez RM. Potential contribution of plants bioactive in ruminant productive performance and their impact on gastrointestinal parasites elimination. Agroforest Syst. 2020; 94(4):1415-1432. https://doi.org/10.1007/ s10457-018-0295-6

83. Hoste H, Torres-Acosta JFJ, SandovalCastro CA, Mueller-Harvey I, Sotiraki S, Louvandini $\mathrm{H}$, et al. Tannin containing legumes as a model for nutraceuticals against digestive parasites in livestock. Vet Parasitol. 2015; 312(1-2):5-17. http:// dx.doi.org/10.1016/j.vetpar.2015.06.026

84. Torres-Fajardo RA, González-Pech PG, Sandoval-Castro CA, Torres-Acosta JFJ. Small ruminant production based on rangelands to optimize animal nutrition and health: Building an interdisciplinary approach to evaluate nutraceutical plants. Animals. 2020; 10(10):1-32. https://doi. org/10.3390/ani10101799 